\title{
Severe Obstructive Sleep Apnea in an young Adult
}

\author{
Fatema Yasmin ${ }^{1 *}$, Rawshan Arra Khanam².
}

\begin{abstract}
:
Obstructive Sleep Apnea (OSA) is a serious common sleep disorder needs life long care. OSA is caused by recurrent upper airway obstruction due to increased upper airway collapsibility during sleep. It is highly associated with obesity with several endocrine and metabolic diseases. Hypothyroidism may cause OSA, also linked with obesity. Obesity is also a risk factor for hypertension. This paper is presenting a case of obstructive sleep apnea with obesity, hypertension and hypothyroidism. A 35 years old male presented with the complaints of excessive day time sleepiness, loud snoring, fragmented sleep, apnea like attack, occasional morning headache. Whole night polysomnography was done and diagnosed as severe obstructive sleep apnea. At therapeutic part of polysomnography, he was treated with BiPAP.
\end{abstract}

Key words: Obstructive sleep apnea (OSA), Obesity, Hypertension, Hypothyroidism.

\section{Introduction:}

Obstructive Sleep Apnea (OSA) is the most common sleep disorder. OSA presents with excessive daytime sleepiness, snoring predisposing to cardiac and accident related morbidity and mortality. $24 \%$ to $62 \%$ elderly people suffers with OSA and peak age is about 55 years ${ }^{1}$. Male sex has three times higher risk of sleep apnea ${ }^{2,3}$. Obesity itself individually increases risk factor for $\mathrm{OSA}^{4,5}$. Only $10 \%$ weight gain increases sleep apnea risk apparently $30 \%{ }^{6}$. BMI $\left(\mathrm{kg} / \mathrm{m}^{2}\right)$ is measured by following WHO guideline ${ }^{7}$. Day time sleepiness was subjectively assessed by Epworth Sleepiness scale. Higher the score indicates greater sleepiness ${ }^{8}$. Neck circumference and higher BMI are the most prominent predictor for OSA. Central fat deposition around the neck and abdomen increases risk factor for $\mathrm{OSA}^{9}$. Overnight polysomnography is Gold standard for diagnosis of apnea, where multiple physiologic parameter during sleep is recorded like EEG, EMG, EOG, ECG, Oronasal airflow and oxygen hemoglobin saturation. Complete cessation of airflow for at least 10 seconds is known as apnea. On the basis of effort to breath during the event said to be Obstructive, Central and Mixed apnea. Figure 1 shows different types of Apnea on the effort to breath in diaphragmatic excursions (middle row) in overnight polysomnography. Top row shows cessation of airflow and bottom row shows oxygen haemoglobin desaturation in central, obstructive and mixed varieties. 30\% reduction of oronasal airflow along with 3\% oxygen hemoglobin desaturation is called hypopnea. Severity of sleep apnea indicated by Apnea Hypopnea index (AHI) which is total number of Apnea Hypopnea per hour of sleep.

1. Sleep Consultant. Ingenious Pulmo-Fit, Ring Road, Dhaka, Bangladesh.

2. Consultant, Dept. of Respiratory Medicine, United Hospital, Gulshan 2, Dhaka.

*Corresponding Author:

Dr. FatemaYasmin

MBBS, MD

Sleep Consultant.

Ingenious Pulmo-Fit, Dhaka, Bangladesh.

Email:ms.yasminbm14@gmail.com
OSA severity is defined as mild for AHI $\geq 5$ and $<15$ and moderate for $\mathrm{AHI} \geq 15$ and $<30$ and severe for $\mathrm{AHI} \geq 30 / \mathrm{hr}^{10}$.

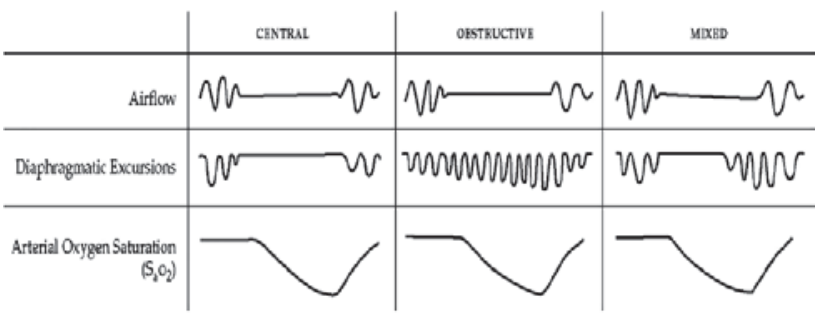

Figure 1

\section{Case report:}

A 35 years old male, resident of Dhanmondi, Dhaka, lawyer presented with the complaints of Excessive day time sleepiness, loud snoring, fragmented sleep, apnea like attack \& occassional morning headache. He is ex-smoker and occasional alcohol consumer (1-2 pegs/week).

On examination, his height was $162 \mathrm{~cm}$ and weight was $82 \mathrm{~kg}$ and BMI was 31 . His neck circumferrence was $43 \mathrm{~cm}$, waist circumferrence was $109 \mathrm{~cm}$ and blood pressure was 130/80 $\mathrm{mmHg}$. He is hypertensive, non diabetic and known case of hypothyroidism for last two years. His ECG shows anterolateral ischemia. His echocardiogram showed good LV and RV systolic function, no regional wall motion abnormality and ejection fraction was $65 \%$ \& PASP $24 \mathrm{~m} \mathrm{~m} \mathrm{Hg}$. His daytime sleepiness score measured by Epwoth sleepiness scale was 17 and his mallapati score was 4 . He regularly takes antihypertensive medication (beta blocker) and also levothyroxin $50 \mathrm{mcg}$.

Nocturnal Polysomnography showed 303 obstructive Apnea, no central Apnea and no mixed Apnea,Total 303 Apnea and 10 Hypoapneas and Apnea Hypoapnea Index was 68.4. His lowest desaturation was 78 of 28.5 seconds duration. His desaturation Index was 66 with 195 times of desaturation during his diagnostic hours of sleep. He was diagnosed as a case of severe OSA.

At the therapeutic procedure, $\mathrm{He}$ was tritrated with CPAP initially, later on BiPAP with oronasal mask at IPAP $11 \mathrm{~cm}$ 
Bangladesh Crit Care J September 2019; 7 (2): 109-110

$\mathrm{H}_{2} \mathrm{O}$ pressure and EPAP $6 \mathrm{~cm} \mathrm{H}_{2} \mathrm{O}$ pressure. His baseline saturation was maintained with Apnea Hypoapnea Index reduced to 0.6 .

\section{Discussion:}

Obstructive sleep apnea presents recurrent closure of upper airway along with oxygen desaturation during sleep. Several studies show that there is an association present between obstructive sleep apnea with other clinical presentation. Obesity, Hypertension and hypothyroidism have individual role on OSA. $40 \%$ moderately obese patients suffer from $\mathrm{OSA}^{4}$. In addition, OSA strongly contributes to obesity related hypertension due to sympathetic overactivity, metabolic and neuroendocrine abnormalities are involved ${ }^{11,12}$. Hypoxia have direct adverse role on cardiac electrical activity which results in sympathetic vasoconstriction and raised blood pressure ${ }^{12}$. Although this patient has normal echocardiography, but pulmonary hypertension is common in OSA. About $12 \%-34 \%$ OSA patients have pulmonary hypertension due to nocturnal intermittent hypoxia which aggravates precapillary pulmonary hypertension ${ }^{13,14}$. About $25 \%$ to $80 \%$ OSA patients have hypothyroidism. Similar contributing factors are responsible for occurrance of OSA in hypothyroidism. Deposition of mucopolysaccharides in upper airway, decreased ventilatory reponse in upper airway and also obesity have the etiological relationship for the of OSA in hypothyroidism ${ }^{15,16}$.

Positive Airway Pressure (PAP) is the treatment choice for mild, moderate and severe OSA. PAP is an effective treatment option for reduction of AHI also improving daytime sleepiness, sleep related quality of life. PAP device is used by nasal, oral and oronasal mask. Three different modes of PAP devices are available for management of OSA like CPAP, BiPAP and $\mathrm{APAP}^{17}$. Hypothyroism with obesity presented with OSA should be treated with overnight PAP therapy even after remission of signs of hypothyroidism ${ }^{18}$.

\section{References:}

1. BixlerE. O, Vgontazs A. N, Lin M. H, CalhounL. S, Vela-Bueno V, Kales A. Excessive daytime sleepiness in a general population sample: the role of sleep apnea, age, Obesity, Diabetes and depression. J ClinEndcrinolMetab 2005; 90(8): 4510-4515.

2. Young T, Palta M, Demsey J, Skatrud J, Weber S, Badr S. The occurrence of sleep- disordered breathingamong middle-aged adults. N Eng J Med.1993;328(17): 1230-1235.
3. Bixler E. O, Vgontazs A. N, Have TT, Kathy T, Kales A. Effects of age on Sleep Apnea in men. Am J RespirCrit Care Med. 1998; 157(1): 144-148.

4. Punjabi NM, Sorkin JD, Katzel LI, Goldberg AP, Schwartz AR, Smith PL. Sleep- Disordered breathing and Insulin resistance in middle-aged and overweight men. Am J RespirCrit Care Med. 2002; 165(5): 677-682.

5. Young T, Peppard PE, Taheri S. Excess weight and sleep-disordered breathing. J Appl Physiol. 2005; 99(4): 1592-1599.

6. Peppard PE, Young T, Palta M, Demsey J, Skatrud J. Longitudinal study of moderate weight change and sleep-disordered breathing. JAMA. 2002;284(23):3015-3021.

7. http://www.euro.who.int/en/health-topics/disease-prevention/nutrit ion/a-healthy-lifestyle/body-mass-index-bmi

8. https://www.healthline.com/health/epworth-sleepiness-scale.

9. Guilleminault C, Stoohs R, Kim YD, Chervin R, Black J,Clerk A. Upper airway sleep-disordered breathing in women. Ann Intern Med.1995;122(7):493-501.

10. Kushida CA, Littner MR, Morgenthaler T, Alessi CA, Bailey D, Coleman J Jr et al. Practice parameters for the indications fo polysomnography and related procedures: an update for 2005. Sleep. 2005; 28(4):499-521.

11. Wolk R, Shamsuzzaman ASM, SomersVK. Obesity, sleep apnea and hypertension. Hypertension. 2003;42(6):1067-1074.

12. Somers VK, Zavala DC, Mark AL. Influence of ventilation and hypocapnia on sympathetic nerve response to hypoxia in normal humans. J Appl Physiol.1989;67(5):2095-2100.

13. Podzus T, Bauer W, Mayer J. Sleep and pulmonary hypertension. Klin Wochenchr.1986:64:131-134.

14. Chaouat A, Weitzenblum E, Krieger J, Oswald M, Kessler R. Pulmonary hemodynamics in the obstructive sleep apnea syndrome. Results in 220 consecutive patients. Chest. 1996 ; 109(2):380-6.

15. Lin C, TsanK, Chen P. The relationship between sleep apnea and hypothyroidism. Chest. 1994; 102(6): 1663-1667.

16. Grunstein RR, Sullivan CE. Sleep apnea and hypothyroidism: mechanism and management. Am. J.Med.1988;85(6):775-779.

17. Epstein LJ, Kristo D, StrollonPJ, Friedman N, Malhotra A, Patil SP et al. Clinical guideline for evalution. Management and long-term care of obstructive sleep apnea in adults. J Clin Sleep Med.2009;5(3):263-276.

18. Rosenow F, McCarthy V, Caruso AC. Sleep apnea in endocrine diseases. J Sleep Res.1998; (7)3-11. 